\title{
A retrospective study of 296 cases of intra uterine fetal deaths at a tertiary care centre
}

\author{
Neetu Singh*, Kiran Pandey, Neena Gupta, A K Arya, Charu Pratap, Reshika Naik
}

Department of Obstetrics and Gynecology, G.S.V.M. Medical College, Kanpur-208002 (U.P.), India

Received: 1 February 2013

Accepted: 22 March 2013

\author{
*Correspondence: \\ Dr. Neetu Singh \\ E-mail: drneetusingh73@gmail.com
}

(C) 2013 Singh N et al. This is an open-access article distributed under the terms of the Creative Commons Attribution License, which permits unrestricted use, distribution, and reproduction in any medium, provided the original work is properly cited.

\begin{abstract}
Background: To identify the risk factors and to streamline preventive and management protocols for IUD.

Methods: This was a retrospective study from January 2011 to December 2012 which was conducted at G.S.V.M. Medical College, Kanpur. IUD was defined as fetal death beyond 20 weeks of gestation and/or birth weight $\geq 500 \mathrm{~g}$. Maternal and fetal records were analysed. Mode of delivery and associated complications were studied.

Results: Total number of deliveries were 7310.Incidence of IUD at our centre was 40 per $1000.55 .73 \%$ were antepartum and $11.06 \%$ were intra partum. In $33.44 \%$ cases, no causes were identified. Among the identifiable causes, very severe anemia $(16.55 \%)$ and hypertensive disorders $(10.81 \%)$ were most common followed by placental causes (12.16\%).Congenital malformations were responsible for $9.45 \%$ cases .Induction was done in 151 patients, 111 patients had spontaneous onset of labour and caesarean section was done in 34 patients. The most devastating complication of IUD was DIC found in 14 patients $(3.71 \%)$.

Conclusions: The present study is an effort to compile a profile of maternal, fetal and placental causes culminating to IUD at our centre. This emphasizes the importance of proper antenatal care and identification of risk factors and its treatment. Institutional deliveries should be promoted to prevent intrapartum fetal deaths .A substantial number of IUD are still labeled as unexplained, hence cannot be prevented. Decrease in the incidence of IUD would significantly reduce the perinatal mortality.
\end{abstract}

Keywords: Intrauterine fetal death (IUD), Unexplained fetal death, Disseminated intravascular coagulation (DIC)

\section{INTRODUCTION}

Intrauterine fetal death (IUD) is a distressing situation for the caregiver and a traumatic event for the family. IUD definition includes antepartum deaths beyond 20 weeks of gestation or birth weight $\geq 500 \mathrm{gm}$ (WHO). More than 3.2 million stillbirths occur globally each year, yet stillbirths are largely invisible in global health indicators, policies and programmes. ${ }^{1}$ Still birth rate in India is 9 per 1000 total births. ${ }^{2}$ Perinatal mortality includes number of stillbirths in the first week of life per 1000 live births(WHO). It is a major marker to assess the quality of health care delivery. The Perinatal Mortality Surveillance Report [CEMACE, 2011] defines stillbirth as a baby delivered without signs of life after
24 completed weeks of pregnancy. This definition was accepted by the Royal College of Obstetricians and Gynecologists in their 2010 Green-top Guideline.

Intrauterine fetal death is a significant contributor to perinatal mortality in developing countries although improved antenatal care, advanced techniques of perinatal diagnosis and better intrapartum monitoring has reduced the incidence. Intrauterine fetal death may be antepartum or intrapartum. Antepartum fetal deaths are associated with several maternal, placental or fetal factors. Hypertensive disorders of pregnancy, anaemia, obesity, diabetes, high parity, advanced maternal age are well recognized maternal factors whereas congenital anomalies, intrauterine growth retardation are important fetal factors. 
Placental causes include abruption and antepartum hemorrhage. Intrapartum fetal death is usually the result of fetal distress and / or obstructed labour and reflects poor quality of clinical care. Cord complications include cord prolapse, tight cord around neck and true knot.

Our study was carried out with the aim of identifying epidemiology of intrauterine deaths and its risk factors and to streamline the preventive and management protocols.

\section{METHODS}

This was a retrospective study from January 2011 to December, 2012 which was conducted at Upper India Sugar Exchange Maternity Hospital, G.S.V.M. Medical College, Kanpur, India. Total number of deliveries during this period was 7310. Among this, total number of IUD including both ante and intra partum deaths were 296. Records were thoroughly analysed with respect to age, parity, gestational age, associated complicating factors like hypertensive disorders of pregnancy, diabetes, Rh isoimmunization, severe anaemia, history of IUD in previous pregnancy. Fetal characteristics were studied with respect to sex, birth weight, gross congenital anomalies. Risk factors related to placenta and cord (true knot, cord prolapse and tight cord around neck)were also analysed. Mode of delivery associated complications and co-morbidities were also studied. Transabdominal USG was done to confirm IUD. Laboratory investigations were studied.

\section{RESULTS}

We observed that $25 \%$ of IUDs were primipara and $24.32 \%$ patients were grand multipara but when the causes among primipara and grand multipara were analysed, placenta previa was common in grand

Table 1: Maternal characteristics.

\begin{tabular}{|c|c|c|c|c|}
\hline S. No. & Maternal Character & & No. & Percentage \\
\hline \multirow{5}{*}{1} & \multirow{5}{*}{ Maternal age (years) } & $<20$ & 56 & $18.91 \%$ \\
\hline & & $21-25$ & 76 & $25.67 \%$ \\
\hline & & $26-30$ & 80 & $27.02 \%$ \\
\hline & & $31-35$ & 54 & $18.24 \%$ \\
\hline & & $>35$ & 30 & $10.13 \%$ \\
\hline \multirow{5}{*}{2} & \multirow{5}{*}{ Parity } & $P_{1}$ & 74 & $25 \%$ \\
\hline & & $\mathrm{P}_{2}$ & 52 & $17.56 \%$ \\
\hline & & $\mathrm{P}_{3}$ & 56 & $18.91 \%$ \\
\hline & & $\mathrm{P}_{4}$ & 42 & $14.18 \%$ \\
\hline & & $\geq \mathrm{P}_{5}$ & 72 & $24.32 \%$ \\
\hline \multirow{4}{*}{3} & \multirow{4}{*}{$\operatorname{BMI}\left(\mathrm{kg} / \mathrm{m}^{2}\right)$} & $<18.5$ & 56 & $18.91 \%$ \\
\hline & & $18.5-24.9$ & 204 & $68.91 \%$ \\
\hline & & $25-29.9$ & 24 & $8.10 \%$ \\
\hline & & $>30$ & 12 & $4.05 \%$ \\
\hline \multirow{4}{*}{4} & \multirow{4}{*}{ Gestational age } & $<34$ week & 44 & $14.86 \%$ \\
\hline & & 34-37week & 48 & $16.21 \%$ \\
\hline & & 37-40week & 190 & $64.18 \%$ \\
\hline & & $>40$ weeks & 14 & $4.72 \%$ \\
\hline
\end{tabular}

Table 2: Fetal characteristics.

\begin{tabular}{|lllll|}
\hline S. No & Fetal Characteristics & No. & Percentage \\
\hline \multirow{3}{*}{1} & & $0.5-0.99$ & 6 & $2.02 \%$ \\
\cline { 3 - 5 } & \multirow{3}{*}{ Fetal weight $(\mathrm{kg})$} & $1.0-1.49$ & 72 & $24.32 \%$ \\
\cline { 3 - 5 } & & $1.5-1.99$ & 42 & $14.18 \%$ \\
\cline { 3 - 5 } & & $2.0-2.49$ & 89 & $30.06 \%$ \\
\cline { 3 - 5 } & \multirow{2}{*}{ Fetal sex } & $2.5-2.99$ & 51 & $17.22 \%$ \\
\cline { 3 - 5 } & & Male sex & 160 & $54.05 \%$ \\
\hline \multirow{2}{*}{2} & \multirow{2}{*}{3} & Female sex & 136 & $45.94 \%$ \\
\hline \multirow{2}{*}{3} & Gross features & Non-macerated & 198 & $66.89 \%$ \\
\cline { 3 - 5 } & & Macerated & 96 & $32.43 \%$ \\
\hline
\end{tabular}


Table 3: Causal factors.

\begin{tabular}{|c|c|c|c|c|}
\hline S. No. & \multicolumn{2}{|l|}{ Causal Factors } & No. & Percentage \\
\hline \multicolumn{5}{|c|}{ I. Antepartum (n=165) } \\
\hline \multirow{4}{*}{1} & \multirow{4}{*}{ Maternal n = $97(32.37 \%)$} & Very severe anaemia & 49 & $16.55 \%$ \\
\hline & & $\begin{array}{l}\text { Hypertensive disorders of } \\
\text { pregnancy }\end{array}$ & 32 & $10.81 \%$ \\
\hline & & History of previous IUD & 12 & $4.05 \%$ \\
\hline & & $\begin{array}{l}\text { Gestational Diabetes } \\
\text { mellitus }\end{array}$ & 4 & $1.35 \%$ \\
\hline \multirow{2}{*}{2} & \multirow{2}{*}{ Fetal $n=32(11.06 \%)$} & Congenital malformations & 28 & $9.45 \%$ \\
\hline & & Rh Incompatibility & 4 & $1.35 \%$ \\
\hline \multirow{4}{*}{3} & \multirow{4}{*}{ Placental $(n=36)(12.16 \%)$} & Abruptio & 20 & $6.75 \%$ \\
\hline & & Placenta previa & 10 & $3.37 \%$ \\
\hline & & IUGR & 4 & $1.35 \%$ \\
\hline & & Post term pregnancy & 2 & $0.67 \%$ \\
\hline \multicolumn{5}{|c|}{ II. Intrapartum (n=32) (11.06\%) } \\
\hline 1 & \multicolumn{2}{|l|}{ Obstructed labour } & 18 & $6.08 \%$ \\
\hline 2 & \multicolumn{2}{|c|}{ Cord prolapse } & 8 & $2.7 \%$ \\
\hline 3 & \multicolumn{2}{|c|}{ Tight cord around neck/ true knot } & 6 & $2.02 \%$ \\
\hline
\end{tabular}

Table 4: Mode of Delivery.

\begin{tabular}{|llll|}
\hline S. No. & Mode of delivery & No. & Percentage \\
\hline I & Induction (n=151) $\mathbf{5 1 . 0 1 \%}$ leading to & \\
\hline & 1. Normal vaginal delivery & 145 & $96.02 \%$ \\
\hline & 2. Instrumental deliveries & 6 & $1.35 \%$ \\
\hline II & Spontaneous labour (n=111) $\mathbf{3 7 . 5 \%}$ leading to & 107 & $96.39 \%$ \\
\hline & 1. NVD & 4 & $3.60 \%$ \\
\hline & 2. Instrumental & $\mathbf{3 4}$ & $\mathbf{1 1 . 4 8 \%}$ \\
\hline III & LSCS* & \multicolumn{2}{|c|}{} \\
\hline *indications like placenta previa, transverse lie, previous 2 caesarean section, obstructed labour \\
\hline
\end{tabular}

Table 5. Maternal morbidity.

\begin{tabular}{|llll|}
\hline S. No. & Maternal Morbidity & No. & Percentage \\
\hline 1. & Psychological upset & 67 & $22.63 \%$ \\
\hline 2. & Deranged coagulation profile & 14 & $4.72 \%$ \\
\hline 3. & Puerperal Sepsis & 5 & $1.68 \%$ \\
\hline 4. & Hospital stay $>7$ days & 28 & $9.45 \%$ \\
\hline \multirow{2}{*}{5.} & Blood transfusion & 156 & $52.70 \%$ \\
\cline { 2 - 4 } & $\geq 1$ units & 74 & $25 \%$ \\
\cline { 2 - 4 } & $\geq 2$ units & 52 & $17.56 \%$ \\
\hline 6. & PPH units & 30 & $10.13 \%$ \\
\hline
\end{tabular}

multipara but in primipara, causes were mainly unexplained. Majority of the patients $(68.91 \%)$ were in the normal BMI category and belonged to age groups $21-30$ years i.e. the period of maximum reproductibility. It was observed that majority of IUD foetuses $(64.18 \%)$ were from 37 to 40 weeks whereas preterm intra-uterine deaths accounted for $31.07 \%$ of total IUDs.

It was observed that $44.24 \%$ of dead fetus weighed from 1.5 to $2.49 \mathrm{~kg}$. Among the IUD fetuses, male sex was found to be significantly higher $(54.05 \%)$ as compared to female sex (45.94\%).In our study $66.89 \%$ fetuses 
were non macerated as compared to $32.43 \%$ macerated fetuses.

Among maternal factors, very severe anemia accounted for $16.55 \%$ IUDs and hypertensive disorders of pregnancy caused $10.81 \%$ of IUD. Fetal factors most commonly found in our study were congenital malformations (9.45\%) and Rh incompatibility (1.35\%). Among the placental factors, $6.75 \%$ were due to abruption and $3.37 \%$ IUDS were due to placenta previa. Obstructed labour $(6.08 \%)$ was the major intrapartum factor. In $33.44 \%$ cases of IUD no cause was identified. Out of 296 IUDs, 151 patients $(51.01 \%)$ needed induction and $111(37.5 \%)$ had spontaneous onset of labour.

Most common morbidity encountered in patients with IUD was psychological upset seen in $22.63 \%$ patients. The most dreaded complication of IUD requiring intensive care unit admission was disseminated intravascular coagulation encountered in $4.72 \%$ of patients. There were 2 mortalities which were due to congestive cardiac failure and disseminated intravascular coagulation.

\section{DISCUSSION}

Incidence of IUD at our centre was found to be 40 per 1000 which is in accordance to study conducted by Maleckiene L in Lithuania. ${ }^{3}$ Out of 296 cases, 32 cases were booked $(10.81 \%)$ and 264 cases were unbooked $(89.18 \%)$. Patients were admitted at our centre with chief complaints of loss of fetal movements (90\%), pain abdomen (6\%) and bleeding per vaginum (4\%).

In our study majority of women were in the age group 20-30 years with normal BMI. This is in contrast to other studies where advanced maternal age and obesity were associated with higher risk of IUD. ${ }^{4,5,6}$ This is because pregnancy in extremes of age group and obesity in reproductive age group is uncommon in our setup.

In our study $25 \%$ patients were primipara, $24.32 \%$ were grand multipara and parity of 2 to 4 were $50.65 \%$ which was in accordance with study conducted by Tariq who also found that parity also had no association with IUD.

In our study, cause of intrauterine fetal death was identifiable in $66.56 \%$ fetuses which included both antepartum as well as intrapartum deaths. In $33.44 \%$ cases, no cause for IUD could be identified.

Antepartum IUD were caused by maternal, fetal and placental factors. Among the maternal factors very severe anemia i.e. $\mathrm{Hb}-4 \mathrm{gm} / \mathrm{dl}$ and hypertensive disorders of pregnancy were associated with significant number of fetal deaths at our centre. This was observed because our centre is tertiary care centre where patients were referred from other centres with these complications and majority of patients were unbooked and did not receive any antenatal care.
Hypertension as a leading cause of IUD was also seen in several other studies. ${ }^{7,8}$ In our study diabetes was found to be associated in $1.35 \%$ cases which was in contrast to study conducted by Gunton where diabetes came out to be the major factor for IUD. ${ }^{9}$

A past history of intrauterine fetal death indicates some subclinical genetic or chromosomal problem which can recur in future pregnancies. In our study history of previous IUD was seen in $4.05 \%$ cases. $^{10}$

Among the fetal causes, major congenital anomalies accounted for $9.45 \%$ cases, out of which 9 had hydrocephalous, 8 had neural tube defects, 7 had anencephaly, 2 cases were gastroschisis, one case of bilateral renal agenesis and one had congenital cardiac disease.

This was in contrast to the study conducted by Tariq where congenital malformations accounted for $25.2 \%$ cases of IUD. ${ }^{11}$ Neural tube defects emerged as the major congenital anomaly responsible for IUD in our set up. This may be due to the lack of folic acid supplementation in periconceptional period.

$\mathrm{Rh}$ isoimmunization was reported in $1.35 \%$ of IUD in our study which was in accordance with the study by Samadi et al who reported $4.7 \%$ incidence. $^{12}$

Male fetus was more vulnerable to fetal death $(54.05 \%)$ which was in accordance to study conducted by Zhang. ${ }^{13}$

It was noticed that most of our fetuses were lost at 37 weeks and beyond. It may indicate that uterine conditions become hostile at this phase and therefore it is recommended to apply closer surveillance at 37 weeks and beyond, so that fetuses will not be lost at that critical period. ${ }^{4}$ The critical peak at which fetuses were lost is variable in theliterature. ${ }^{7,8,10,14}$ This may indicate that predisposing pathology for IUFD is different in different communities.

Among the placental causes, $6.75 \%$ was due to abruption and $3.37 \%$ was due to placenta previa. This is in accordance to study conducted by Jahanfar. ${ }^{15}$

Intrapartum fetal death accounted for $11.06 \%$ of fetal deaths. Among intrapartum complications, obstructed labour leading to IUD was common in our study $(6.08 \%)$. These are rarely seen in developed countries. This is due to patients' ignorance and lack of well equipped health care delivery system at grass root level.

Cord complications like cord prolapse and true knot was seen in $4.72 \%$ of patients in our study which was in contrast to study conducted by Tariq ${ }^{11}$ where cord accidents accounted for $13.3 \%$ IUDs. 
Unexplained stillbirths account for 25 to $60 \%$ of total stillbirths, ${ }^{16,17}$ whereas in our study it accounted for $33.44 \%$ of deaths. The number of unexplained still births has been sharply reduced by the increasing resort of necropsy in western countries. The absence of necropsy in our series seriously inhibited on diagnostic accuracy. This wide variation in rates is both a reflection of the classification systems used to define stillbirth and the extent of postmortem assessment performed in each case.

Onset of labour was spontaneous in $37.5 \%$ cases while $51.01 \%$ patients needed induction. Induction was done with misoprostol and its dose varied according to the gestation age.11.48\% patients were directly taken for caesarean section for indications like placenta previa, transverse lie, previous 2 caesarean section and obstructed labour.

In our study, $4.7 \%$ patients had DIC and $1.68 \%$ patients had puerperal sepsis. These patients came with prolonged retention of dead fetus. When a baby dies before birth, the options for care are either to wait for labour to start spontaneously or to induce labour. In majority of women $(90 \%)$ labour begins within three weeks of IUD, but if labour does not begin, there is a risk of developing a disseminated intravascular coagulopathy (DIC), as well as intra-uterine infection if the membranes are ruptured. ${ }^{18}$ Other disadvantage of a long interval between fetal death and birth relate to greater emotional distress. Psychological upset was seen in $22.56 \%$ of patients. ${ }^{19}$ A wide range of short and longterm negative outcomes for parents has been reported as a result of infant death. ${ }^{20}$

Blood transfusion was required in $53 \%$ of cases because majority of the patients were severely anaemic. Duration of hospital stay $>7$ days was seen in $9.45 \%$ patients. It was seen in patients who had some complication of IUD.

Although very severe anemia, hypertensive disorders of pregnancy, placental disorders, lethal congenital anomalies and cord complications are important indicative factors of still birth in our population yet more studies are required to find the causes of unexplained still birth which constitutes a substantial proportion of perinatal mortality.

\section{CONCLUSION}

The purpose of counting IUD is to understand the contributory factors and to seek ways of avoiding recurrence by proper antenatal care and early diagnosis of complications and its adequate management. Clinical assessment and evaluation is recommended to assess maternal wellbeing and to determine the cause of death, the chance of recurrence and of avoiding further pregnancy complications (RCOG, 2010 guidelines).
The modifiable maternal risk factors such as hypertension, severe anaemia and diabetes control can prevent intrauterine fetal death. First and second trimester ultrasound evaluation may be helpful in ruling out congenital malformations and placental disorders which are also implicated in intrauterine fetal death. Better intrapartum fetal monitoring for high risk cases can lead to prevention of IUFD. In conclusion, the associated risk factors in our community seem to be preventable. We should pay attention to health education with emphasis on antenatal care and the benefit of regular attendance, improved periconceptional environment, nutrition, micronutrient status especially iron and folic acid intake. Identification of high risk cases and their timely referral to higher centres may save the baby. Patient compliance is important in reducing most of these preventable fetal losses.

Women with a history of IUFD should attend a consultant-led hospital-based antenatal clinic in their next pregnancy and undergo increased antenatal surveillance. Future research should focus on improved means of clinical assessment of fetal well being and defining pathophysiological pathways leading to still birth associated with maternal disease. Parents have the greatest stake of all in the wellbeing of their baby, and must be part of the drive to reduce stillbirth. We should let the life of every mother and baby count.

\section{ACKNOWLEDGEMENTS}

The authors appreciate all the researches whose articles were included in the study for the comparative analysis of all the factors which contributed for IUD.

Funding: No funding sources

Competing interests: None declared

Ethical approval: Not required

\section{REFERENCES}

1. Stanton C, Lawn JE, Rahman H, Wilczynka-Ketende K, Hill K. Stillbirth rates: delivering estimates in 190 countries. Lancet 2006;367:1487-94.

2. Census of India vital statistics, figures at a glance India 2003 Ministry of home affairs, Govt. of India.

3. Maleckiene L, Nadisauskiene R, Stankeviciene J, Cizauskas A, Bergstrom S. A case reference study on fetal bacteremia and late fetal death of unknown etiology in Lithuania. Acta Obstet Gynecol Scand 2000;79:1069-74.

4. Canterino JC, Ananth CV, Smulian J, Harrigan JT, Vintezileos AM. Maternal age and risk of fetal death in singleton gestations: USA, 1995-2000. J Matern Fetal Neonatal Med 2004;15:193-7.

5. Nohr EA, Bech BH, Davies MJ, Frydenberg M, Henriksen TB, Olsen J. Pre pregnancy obesity and fetal death: a study within the Danish National Birth Cohort. Obstet Gynecol 2005;106:250-9. 
6. Stephansson O, Dickman PW, Johansson AL, Cnattingius S. Maternal weight, pregnancy weight gain, and the risk of antepartum stillbirth. Am J Obstet Gynecol 2001;184:463-9.

7. Smulian JC, Ananth CV, Vintzileos AM, Scorza, Knuppel RA, et al. Fetal death in The United States. Influence of high risk conditions and implication for management. Obstet Gynecol 2002, 100:1183-91.

8. Archibong EL, Sobande AA, Asindi AA. Antenatal intrauterine fetal death; A prospective study in a tertiary hospital in western Saudi Arabia. J Obstet Gynaecol 2003;23:170-3.

9. Gunton JE. Outcome of pregnancies complicated by pregestational diabetes mellitus. J Obstet Gynecol 2000;40:38-43.

10. Shankar M, Navti O, Amu O, Konjel JC. Assessment of still birth risk and associated risk factors in a tertiary hospital. J Obstet Gynaecol 2002;22:34-8.

11. Khashoggi TU. Epidemiology of intrauterine fetal deaths in Saudi Arabia: kkuh experience. Biomedical Research 2005;16:59-64.

12. Samadi R, Miller D, Settlage, et al. Massive fetomaternal hemorrhage and fetal death: Is it predictable? Am J Obstet Gynecol 1996;174:391.

13. Zhang J, Klebanoff MA. Small-for-GestationalAge Infants and Risk of fetal death in subsequent pregnancies. N Eng J Med 2004;350:754-6.
14. Froen JF, Arnestad M, Frey K, Verge A, Saugstad OD, Stary-pedersen M, et al. Risk factors for sudden intrauterine un-explained fetal death: Epidemiologic characteristic of singleton cases in Oslo, Norway, 1986-1995. Am J Obstet Gynecol 2001;184:649-702.

15. Jahanfar Sh, Ghiyasi P, Haghani H. Risk factors related to intra uterine fetal death in Iran, A casecontrol study. Shiraz E-Medical Journal 2005;6:34.

16. Fretts RC. Etiology and prevention of stillbirth. Am J Obstet Gynecol 2005:193:1923-35.

17. Silver RM, Varner MW, Reddy U, Goldenbery R, Pinar H, Conway D, et al. Work-up of stillbirth: a review of the evidence. Am J Obstet Gynecol 2007;5:433-44.

18. Weiner CP. Fetal Death. In: James DK, Steer PJ, Weiner CP, Gonik B editor(s). High risk pregnancy management options. Second Edition. London: WB Saunders, 1999.

19. Fish W. Differences in grief intensity in bereaved parents. Champaigne, IL: Research Press Company, 1986.

20. Hughes P, Riches S. Psychological aspects of perinatal loss. Curr Opin Obstet Gynecol 2003;15:107-11

DOI: $10.5455 / 2320-1770$. ijrcog20130606

Cite this article as: Singh N, Pandey K, Gupta N, Arya AK, Pratap C, Naik R. A retrospective study of 296 cases of intra uterine fetal deaths at a tertiary care centre. Int J Reprod Contracept Obstet Gynecol 2013;2:141-6. 